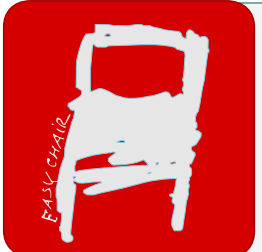

EPiC Series in Health Sciences

CAOS 2017. 17th Annual Meeting of the International

Society for Computer Assisted Orthopaedic Surgery

\title{
CAS guided RFA can be a safe and effective treatment in OFD like adamantinoma in a child.
}

PC Jutte, JJW Ploegmakers

Introduction

Osteo Fibrous Dysplasia (OFD) like adamantinoma is considered a benign condition that is usually located in the tibia. The condition is mainly found in children and has an association with classical adamantinoma, a malignancy of bone. The preferred treatment is surgical.

The lesion often requires a segment resection to eradicate the tumor as the margins of healthy bone are often too small to save continuity of bone and the lesion is located in the cortical area. Segmental resection in a young child is associated with a high complication rate like fractures in hemicortical resection in 6 of 22 patients [1]. Ingrowth of the reconstruction allograft can be better if CAS is used for a better fit [2], however in general the reconstruction with allograft has high complication rates of up to $40 \%$ pseudarthrosis, infection and fracture [3]. To avoid these complications it would be ideal to spare the continuity of tibial bone by treating the tumor in situ. Radiofrequency Ablation (RFA) may be a safe and reliable option for treatment of OFD like adamantinoma, without vital tumor spill and without the need for reconstruction. The RF antenna creates a local field of high temperature to ensure protein denaturation and apoptosis in a planned field. To ensure accurate local tumor ablation, planning, guidance and monitoring of antenna placement, as well as execution of the local tumor ablation are performed with computer assisted surgery (CAS).

Materials and methods

Three young patients with OFD like adamantinoma of the tibia were treated with CAS guided mini open intra-operative RFA in our institution. 
CAS guided RFA can be a safe and effective treatment in OFD ... Paul Jutte and Joris Ploegmakers

Results

Because of the heat generation and the proximity of the skin we performed the procedures mini open to protect the skin from burn wounds. In all three patients successful local tumor ablation was achieved without complications. At baseline MRI there was no sign of residual tumor activity in either patient.

Discussion

Follow-up is too short and number of treated patients is too low to draw definitive conclusions yet.

What we can say is that there is considerable less damage done to the patient if compared to classical open surgery like segmental resection or hemicortical resection.

Another great advantage is that it allows accurate local tumor ablation without tumor spill and it does not prohibit a local resection as a plan b in case of viable local tumor tissue visible on MRI. It allows classical treatment in case of insufficient ablation.

Modeling, fusion of planning and images as well as segmentation is feasible and reliable in predicting the ablation area in this small tumor size.

Local tumor ablation with RFA has the potential to become a safe and effective treatment alternative in OFD like adamantinoma in a child.

\section{References}

1 Deijkers RL, Bloem RM, Hogendoorn PC, Verlaan JJ, Kroon HM, Taminiau AH. Hemicortical allograft reconstruction after resection of low-grade malignant bone tumours. Bone \& Joint Journal. 2002 Sep 1;84(7):1009-14.

2 Gerbers JG, Ooijen PM, Jutte PC. Computer-assisted surgery for allograft shaping in hemicortical resection: a technical note involving 4 cases. Acta orthopaedica. 2013 Jan 1;84(2):224-6.

3 Bus MP, Bramer JA, Schaap GR, Schreuder HW, Jutte PC, van der Geest IC, van de Sande MA, Dijkstra PD. Hemicortical Resection and Inlay Allograft Reconstruction for Primary Bone Tumors. J Bone Joint Surg Am. 2015 May 6;97(9):738-50.

Disclosures:

Non 


\section{Figures:}

1a: OFD like adamantinoma in the proximal tibia in a 15 year old girl.

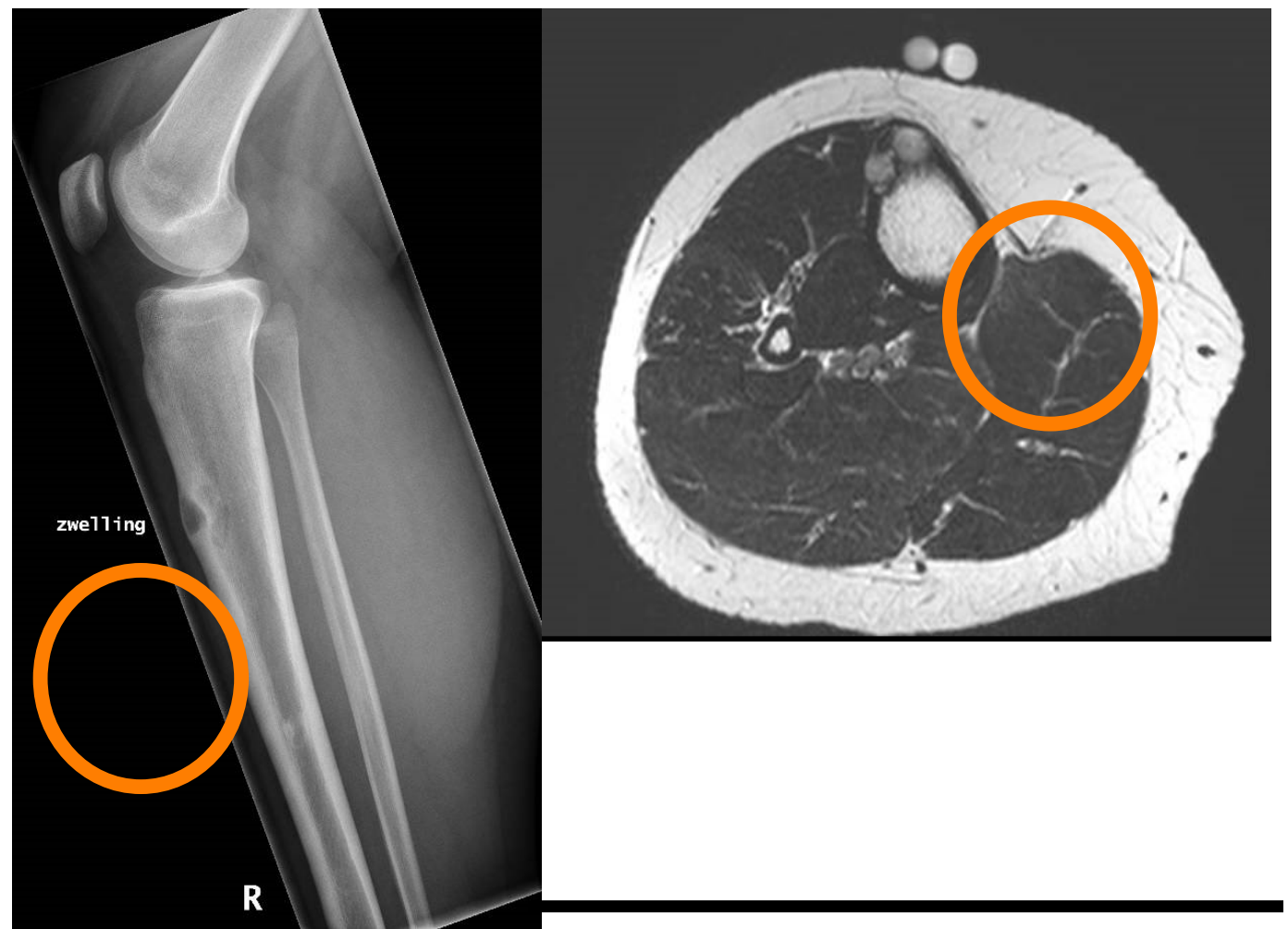

Figure 1b: after RFA there is a halo visible with clear apoptosis in the treated area demonstrated by the lack of contrast uptake in the lesion. 


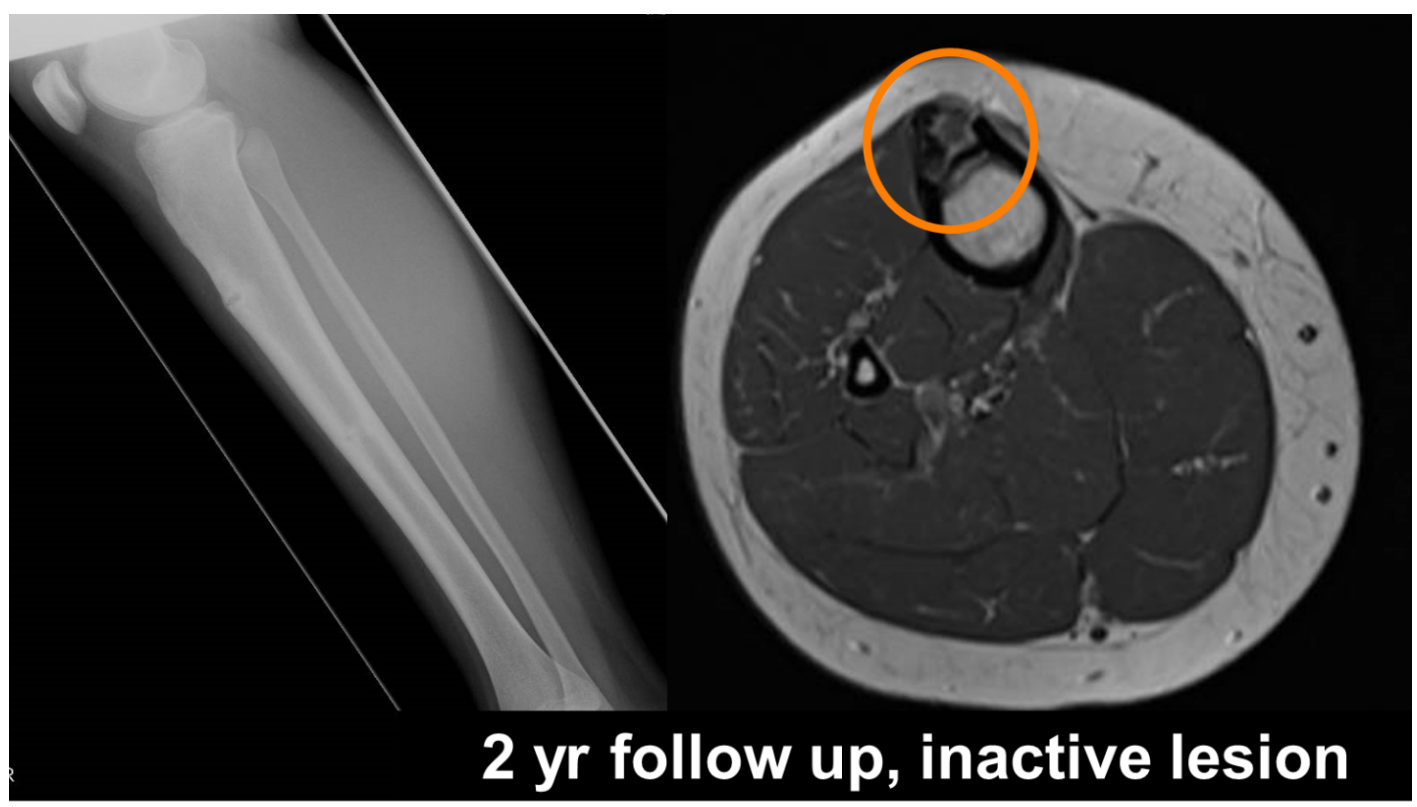

